\title{
TITLE:
}

\section{Ab initio study of stress-induced domain switching in $\mathrm{PbTiO} 3$}

\section{$\operatorname{AUTHOR}(\mathrm{S})$ :}

Shimada, Takahiro; Umeno, Yoshitaka; Kitamura, Takayuki

\section{CITATION:}

Shimada, Takahiro ...[et al]. Ab initio study of stress-induced domain switching in PbTiO3. PHYSICAL REVIEW B 2008, 77 (9): 094105.

\section{ISSUE DATE:}

2008-03

URL:

http://hdl.handle.net/2433/84622

RIGHT:

(C) 2008 The American Physical Society 
PHYSICAL REVIEW B 77, 094105 (2008)

\title{
$A b$ initio study of stress-induced domain switching in $\mathrm{PbTiO}_{3}$
}

\author{
Takahiro Shimada, ${ }^{1, *}$ Yoshitaka Umeno, ${ }^{2}$ and Takayuki Kitamura ${ }^{1}$ \\ ${ }^{1}$ Graduate School of Engineering, Kyoto University, Sakyo-ku, Kyoto 606-8501, Japan \\ ${ }^{2}$ Institute of Industrial Science, The University of Tokyo, Meguro-ku, Tokyo 153-8505, Japan
}

(Received 16 January 2008; published 6 March 2008)

\begin{abstract}
We investigated the atomistic and electronic structure of the $90^{\circ}$ domain wall in $\mathrm{PbTiO}_{3}$ and the fundamental mechanism of domain switching induced by shear stress using first-principles density functional theory calculations within the local density approximation. Under strain-free condition, the magnitude of polarization at the center of the domain wall decreased by $20 \%$ from that of the bulk, and the direction rotated within the transition region of $1.3 \mathrm{~nm}$. Under strain, the applied shear deformation concentrated near the $90^{\circ}$ domain wall, and the domain wall began to migrate in a direction perpendicular to itself after the stress reached the critical magnitude of $152 \mathrm{MPa}$. The migration direction was governed by the shearing direction. During stress-induced domain switching, a $\mathrm{Pb}-\mathrm{O}$ covalent bond at the center of the domain wall broke, and concurrently, another bond on the neighboring $\mathrm{Pb}-\mathrm{O}$ site was formed with a large movement of the $\mathrm{Pb}$ atom. Thus, reconstruction of the $\mathrm{Pb}-\mathrm{O}$ bond was associated with the domain switching.
\end{abstract}

DOI: 10.1103/PhysRevB.77.094105 PACS number(s): 77.80.Dj, 77.80.Fm, 77.84.Dy, 31.15.A-

\section{INTRODUCTION}

Ferroelectric perovskite oxides have attracted great interest because of their promising industrial applications such as nonvolatile random access memories, actuators, sensors, and transducers. ${ }^{1-4}$ Ferroelectric crystals usually consist of domains, each of which has a uniform orientation of spontaneous polarization. These domains are formed through a paraelectric-to-ferroelectric phase transition to minimize the electrostatic energy of the depolarizing fields and the elastic energy of mechanical strains. ${ }^{5} \mathrm{In} \mathrm{PbTiO}_{3}$, which undergoes a single phase transition from the cubic to the tetragonal phase, there are $90^{\circ}$ and $180^{\circ}$ domain walls where the polarization directions of neighboring domains are perpendicular and antiparallel, respectively. ${ }^{6}$ The domain structure plays a significant role in determining both the electrical and mechanical properties of ferroelectrics. For example, the macroscopic spontaneous polarization and the piezoelectric response are strongly affected. ${ }^{3}$ In addition, the kinetics of the domain walls is closely related to a serious problem of polarization fatigue, namely, time-dependent decay in switchable polarization. ${ }^{5}$ Thus, studying the microscopic structure of the domain wall and its switching behavior is essential for understanding the effect on ferroelectricity and piezoelectricity.

In applications such as actuators and transducers, ferroelectrics are subject to not only electrical fields but also mechanical loads. The applied stress or strain often acts as a driving force for the motion of the domain wall. In recent years, Shang and $\operatorname{Tan}^{7}$ have studied the effect of shear stress by means of microindentation tests and revealed that shear stress applied to a $90^{\circ}$ domain wall in $\mathrm{Pb}\left(\mathrm{Mg}_{1 / 3} \mathrm{Nb}_{2 / 3}\right) \mathrm{O}_{3}-\mathrm{PbTiO}_{3}$ (PMN-PT) develops polarization reorientation through a domain-wall movement perpendicular to itself, which is known as stress-induced domain switching. However, the microscopic mechanism of domain switching has not yet been understood.

High-resolution transmission electron microscopy observations ${ }^{8,9}$ have revealed the width of the $90^{\circ}$ domain wall in $\mathrm{PbTiO}_{3}$ to be $1.5 \pm 0.3$ or $1.0 \pm 0.3 \mathrm{~nm}$, which corre- sponds to several lattice spacings. This suggests that a precise atomistic analysis is necessary for the study of stressinduced domain switching. Meyer and Vanderbilt ${ }^{10}$ investigated the $180^{\circ}$ and $90^{\circ}$ domain walls in $\mathrm{PbTiO}_{3}$ using $a b$ initio (first-principles) calculations based on the density functional theory (DFT), ${ }^{11,12}$ which has been validated for the domain-wall structure by comparison with experimental observations.

In this study, we conduct first-principles DFT calculations to investigate the atomic structure of the $90^{\circ}$ domain wall in $\mathrm{PbTiO}_{3}$ and the shear-induced domain switching. This paper is organized as follows. In Sec. II, we describe the details of the simulation method and model. In Sec. III, the domainwall energy and the fully relaxed $90^{\circ}$ domain-wall structure are presented. Furthermore, the response of the domain wall to shear strain and the fundamental process of stress-induced domain switching are discussed from both the atomistic and electronic points of view. Finally, the results are summarized in Sec. IV.

\section{COMPUTATIONAL DETAILS}

\section{A. Simulation method}

$A b$ initio (first-principles) calculations based on the density functional theory with a plane wave basis set are conducted using the Vienna $a b$ initio simulation package (VASP). ${ }^{13,14}$ The pseudopotentials based on the projector augmented wave (PAW) method ${ }^{15}$ include the $\mathrm{O} 2 s$ and $2 p$, the $\mathrm{Ti} 3 s, 3 p, 3 d$, and $4 s$, and the $\mathrm{Pb} 5 d, 6 s$, and $6 p$ electrons in the valence states. The cut-off energy of the plane waves is $500 \mathrm{eV}$. The local density approximation of the CeperleyAlder form ${ }^{16}$ is used to estimate the exchange-correlation energy.

\section{B. Simulation models and procedure}

Figure 1(a) shows the unit cell of tetragonal $\mathrm{PbTiO}_{3}$. Atoms are displaced in the $z$ direction from the symmetrical lattice site with a tetragonal lattice distortion of $c / a$ in the 
(a)


FIG. 1. (Color online) (a) Unit cell of tetragonal $\mathrm{PbTiO}_{3}$. The tetragonality, $c / a$, is exaggerated for clarity. (b) Simulation model of the $90^{\circ}$ domain wall containing $N=12$ perovskite unit cells. The solid box represents the simulation cell. The arrows labeled $P$ indicate the polarization direction.

ferroelectric phase. As a consequence, polarization $P$ emerges along the $z$ axis. The $90^{\circ}$ domain wall is formed on the (101) plane with almost a perpendicular relationship in polarization on both sides of the wall. Figure 1(b) shows a simulation model for the $90^{\circ}$ domain wall. Since the threedimensional periodic boundary condition is applied in the first-principles plane-wave pseudopotential calculations, the simulation cell used in this study contains two $90^{\circ}$ domain walls: DW-A at the edge of the simulation cell and DW-B at the center. The $x$ component of the polarization across the domain wall is in the same direction, while the $z$ component is in the opposite direction, which is a commonly observed head-to-tail-type $90^{\circ}$ domain wall. According to the geometrical requirement, the orthorhombic simulation cell dimensions in the $x, y$, and $z$ directions are set to $N c / \sqrt{1+(c / a)^{2}}, a$, and $a \sqrt{1+(c / a)^{2}}$, respectively, using the theoretical lattice constants of the bulk, $a=0.3867 \mathrm{~nm}$ and $c=0.4034 \mathrm{~nm}(c / a=1.043)$. Here, $N$ denotes the number of perovskite unit cells in the simulation cell, and the model depicted in Fig. 1(b) contains $N=12$ unit cells. For descriptive purposes, we assign the numbers $1 \sim N$ to the $\mathrm{Pb}-\mathrm{Ti}-\mathrm{O}$ type (101) planes. The planes outside the cell in the $-x$ direction are named $-1,-2, \ldots$, which are equivalent to the planes $N, N-1, \ldots$, respectively, because of the periodic boundary condition.

To avoid undesirable influences from neighboring domain walls, the distance between the domain walls should be large enough so that the interaction is negligible. In this study, the appropriate distance is evaluated by checking the convergence of the domain-wall energy and structure of the eight
TABLE I. $90^{\circ}$ domain-wall energy, $E_{\mathrm{DW}}\left(\right.$ in $\left.\mathrm{mJ} / \mathrm{m}^{2}\right)$, as a function of simulation cell size $N$.

\begin{tabular}{ccccccccc}
\hline \hline$N$ & 6 & 8 & 10 & 12 & 14 & 16 & 18 & 20 \\
\hline$E_{\text {DW }}$ & 26.0 & 28.4 & 29.2 & 29.5 & 29.8 & 29.2 & 29.3 & 29.2 \\
\hline \hline
\end{tabular}

simulation models having different $x$ cell dimensions, $6 c / \sqrt{1+(c / a)^{2}}, \quad 8 c / \sqrt{1+(c / a)^{2}}, \quad \ldots, \quad 20 c / \sqrt{1+(c / a)^{2}}$ $(N=6,8, \ldots, 20)$. The $k$-point grids for the Brillouin zone integration selected according to the Monkhorst-Pack scheme $^{17}$ are $2 \times 4 \times 3$ for the $N=6$ and 8 models and $1 \times 4 \times 3$ for the $N \geqslant 10$ models. Atomic positions are relaxed using the conjugate gradient method until all the HellmannFeynman forces are less than $2.5 \times 10^{-3} \mathrm{eV} / \AA$. The glide mirror symmetry across the $z=0$ plane with a translation of half the cell along the $x$ axis is kept during the relaxation. Therefore, DW-A and DW-B are equivalent to each other in the unstrained state.

For the shear simulation, a small shear strain $\Delta \gamma_{x z}$ is applied to the simulation cell step by step [see Fig. 1(b)]. At each strain, atoms are fully relaxed by the same procedure as mentioned above. Note that DW-A and DW-B no longer have the same structure because the glide mirror symmetry is broken under the shear. The strain condition of DW-B is equivalent to that of DW-A sheared in the opposite direction, $-\gamma_{x z}$.

\section{RESULTS AND DISCUSSION}

\section{A. Domain-wall energy and relaxed structure}

The $90^{\circ}$ domain-wall energy $E_{\mathrm{DW}}$ is evaluated by

$$
E_{\mathrm{DW}}=\frac{E_{N}-E_{\mathrm{bulk}} N}{2 S_{\mathrm{DW}}},
$$

where $E_{N}, E_{\mathrm{bulk}}$, and $S_{\mathrm{DW}}$ denote the potential energy of the relaxed simulation model with size $N$, the bulk energy per unit cell, and the cross-sectional area of the $y-z$ plane, respectively. To ensure accuracy, energies of the relaxed structures are recalculated with a denser $1 \times 6 \times 4$ MonkhorstPack $k$-point mesh. The calculated domain-wall energies are tabulated as a function of the simulation cell size in Table I. The domain-wall energy converges at about a cell size of $N=16$, and its magnitude is estimated to be $29 \mathrm{~mJ} / \mathrm{m}^{2}$. This is slightly lower than the $35 \mathrm{~mJ} / \mathrm{m}^{2}$ calculated by Meyer and Vanderbilt ${ }^{10}$ using the Vanderbilt-ultrasoft pseudopotentials $\left(\mathrm{USPP}^{18}\right)$ method. The small deviation might be caused by the difference in methodology because the PAW method implemented in VASP slightly underestimates the ferroelectric state compared with the USPP method: Spontaneous polarization of the $\mathrm{PbTiO}_{3}$ bulk calculated using the Berry phase theory ${ }^{19}$ is $78.6 \mu \mathrm{C} / \mathrm{cm}^{2}$ for PAW and $81.2 \mu \mathrm{C} / \mathrm{cm}^{2}$ for USPP. The $180^{\circ}$ domain-wall energy derived from firstprinciples calculations ${ }^{10,20}$ is 132 or $150 \mathrm{~mJ} / \mathrm{m}^{2}$. These values are much higher than that of the $90^{\circ}$ domain wall. This suggests that the $90^{\circ}$ domain wall is more stable in $\mathrm{PbTiO}_{3}$.

We introduce a local polarization in order to elucidate how the ferroelectric distortions change across the domain 


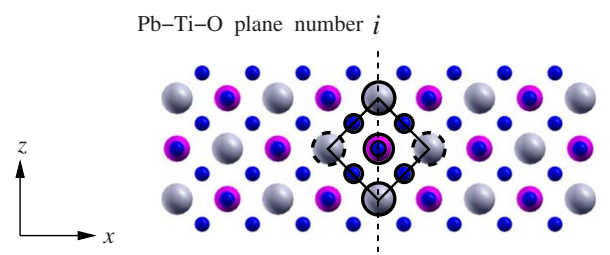

FIG. 2. (Color online) Schematic illustration defining the fictitious unit cell at the $\mathrm{Pb}-\mathrm{Ti}-\mathrm{O}$ plane number $i$. The solid box and circles indicate the unit cell and atoms in the cell, respectively. Atoms are arranged in the ideal cubic positions for clarity.

wall. Here, we define a fictitious "unit cell" at the $\mathrm{Pb}-\mathrm{Ti}-\mathrm{O}$ plane number $i$, as illustrated in Fig. 2 by the solid box, where atoms are represented by solid circles. The $\mathrm{Pb}$ atoms off the $i$ plane are not included. This is the same definition as the "M-centered" choice in Ref. 10, which has been proven to give the finest resolution for the polarization distribution. The local polarization in the unit cell of $i, \mathbf{P}^{(i)}$, is evaluated by

$$
\mathbf{P}^{(i)}=\frac{e}{\Omega_{c}} \sum_{j} w_{j} \mathbf{Z}_{j}^{*} \mathbf{u}_{j},
$$

where $\Omega_{c}, e$, and $\mathbf{u}_{j}$ denote the volume of the unit cell, the electron charge, and the atomic displacement vector from the ideal lattice site of atom $j$, respectively. Index $j$ runs over all atoms in the unit cell $i$. $\mathbf{Z}_{j}^{*}$ is the Born effective charge tensor of the cubic bulk $\mathrm{PbTiO}_{3}$. In this study, we use theoretical values of the Born effective charge tensors calculated by Zhong et al. ${ }^{21}$ Weights $w$ are set to $1 / 4$ for $\mathrm{Pb}, 1$ for Ti, and $1 / 2$ for $\mathrm{O}$. These correspond to the number of unit cells that share the atom. This evaluation has already been validated in Ref. 10. Figure 3 shows distributions of the local polarization calculated for the models with a different simulation cell size $N$. Here, $\phi$ is the polarization direction angle defined as

$$
\phi=\tan ^{-1}\left(P_{z} / P_{x}\right) .
$$

Only the vicinity of DW-A is shown in the figure because DW-B possesses an equivalent structure owing to the symmetry. The local polarization, especially at the -1 and 1 planes, is almost independent of the simulation cell size when $N \geqslant 16$. This indicates again that undesirable interactions between the domain walls are negligible for $N \geqslant 16$.

The transient area of the local polarization is confined to a few layers near the wall. The magnitude of the local polarization at the -1 and 1 planes is about $20 \%$ lower than that of the bulk. This suggests that the misfit strain at the domain wall suppresses ferroelectricity. In the transient region, the polarization direction angle varies by approximately $50^{\circ}$, showing a sharp change in polarization direction from right up to right down. Thus, as a rough estimation, the domainwall dominant region is between planes -3 and 3 , which is evaluated to be $1.3 \mathrm{~nm}$. This is in good agreement with the experimental observations ${ }^{8,9}$ of $1.5 \pm 0.3$ and $1.0 \pm 0.3 \mathrm{~nm}$.

Figure 4 depicts the atomistic configurations and the valence charge density distribution near DW-A projected on the $\mathrm{PbO}-$ and $\mathrm{TiO}_{2}-(010)$ planes of the highly converged simulation model, $N=18$. Electrons are localized between the $\mathrm{Pb}$


FIG. 3. Magnitude of polarization $|\mathbf{P}|$ and polarization direction $\phi$ distributions near DW-A calculated for models with a different simulation cell size $N$.

and $\mathrm{O}$ atoms and between the $\mathrm{Ti}$ and $\mathrm{O}$ atoms. As experimental and theoretical studies have elucidated, ${ }^{22,23}$ these are the covalent bonds constructed in $\mathrm{PbTiO}_{3}$ by hybridizing the $\mathrm{Pb} 6 s$ and $\mathrm{O} 2 p$ states and the Ti $3 d$ and $\mathrm{O} 2 p$ states, respectively. The $\mathrm{Pb}-\mathrm{O}$ and Ti-O covalent bonds across DW-A are indicated by white lines in the figure. On both the $\mathrm{PbO}$ and $\mathrm{TiO}_{2}$ planes, a series of bonds with a "right-down" shape is found on the left side of the domain wall, while the



FIG. 4. (Color online) Atomistic configurations and charge density distributions near DW-A projected on the $\mathrm{PbO}$ - and $\mathrm{TiO}_{2}-(010)$ planes obtained with the simulation cell of $N=18$. The gray, purple, and blue spheres represent $\mathrm{Pb}, \mathrm{Ti}$, and $\mathrm{O}$ atoms, respectively. The $\mathrm{Pb}-\mathrm{O}$ and the Ti-O covalent bonds are drawn with white lines. 


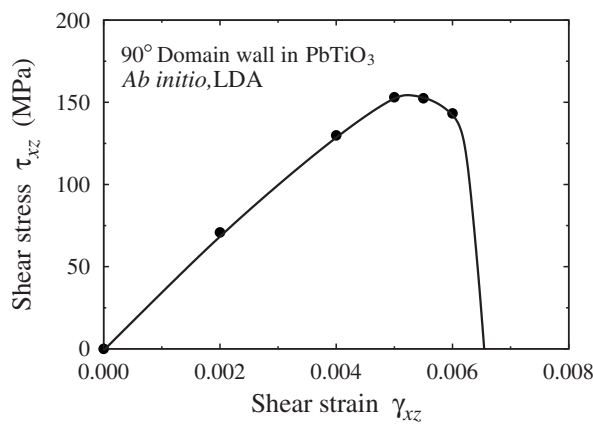

FIG. 5. Shear stress-strain curve calculated with the simulation cell of $N=18$.

"right-up" shape is found on the right side. At the center of the domain wall, the series of bonds on either side are connected smoothly by sharing the $\alpha$ bond for the $\mathrm{PbO}$ plane and the $\beta$ bond for the $\mathrm{TiO}_{2}$ plane. Consequently, a downward convex of the bonding structure is formed at the center. This smooth bond connection contributes to the lower domain-wall energy and the small domain-wall region.

\section{B. Shear deformation and domain switching}

Figure 5 shows the shear stress as a function of the strain in the simulation model of $N=18$.

We also conducted a more accurate simulation using a $1 \times 6 \times 4$ Monkhorst-Pack $k$-point mesh, which showed that the difference in the resulting stress is less than $1 \%$. Thus, the relation shown in Fig. 5 is reliable. The stress varies linearly with strain. Beyond the maximum shear stress, $\tau_{c}$ of $152 \mathrm{MPa}$ at $\gamma_{x z}=0.005$, the stress drops abruptly.

Figure 6 plots changes in distributions of the magnitude and direction of local polarization near DW-A and DW-B under shear deformation. For DW-A, remarkable changes in the local polarization are found between the -1 and 2 planes. This indicates that the shear deformation is localized in the vicinity of the domain wall. The magnitude of the polarization on the -1 plane is enhanced, while that on the 2 plane decreases as the applied shear strain increases. At $\gamma_{x z}=0.007$, where a dramatic decline in shear stress occurs, the polarization minimum, which is initially located between the -1 and 1 planes, shifts from the 1 to the 2 plane. In addition, the polarization direction angle $\phi$ on the 1 plane increases and reaches $23^{\circ}$ at $\gamma_{x z}=0.007$, suggesting that the local polarization rotates from the right-down to right-up direction. As a result, DW-A propagates in the $+x$ direction (the direction normal to the wall) by one interval of the $\mathrm{Pb}-\mathrm{Ti}-\mathrm{O}$ planes. Since this indicates stress-induced domain switching, the maximum stress of $152 \mathrm{MPa}$ is the critical shear stress for domain switching in $\mathrm{PbTiO}_{3}$. For DW-B, the response of the local polarization distribution to the shear strain is quite similar to that of DW-A: the variation of the polarization is concentrated on the 8-10 planes and the polarization on the 9 plane rotates with increasing strain. Thus, DW-B moves from the $9-10$ planes to the $8-9$ (in the $-x$ direction) at the same critical strain of $\gamma_{x z}=0.007$. Note that the strain condition of DW-B is equivalent to that of DW-A subjected to a shear strain in the opposite direction, $-\gamma_{x z}$. The interesting feature found here is that the propagation direction of the domain wall varies with shearing direction. However, the critical



(b) DW-B
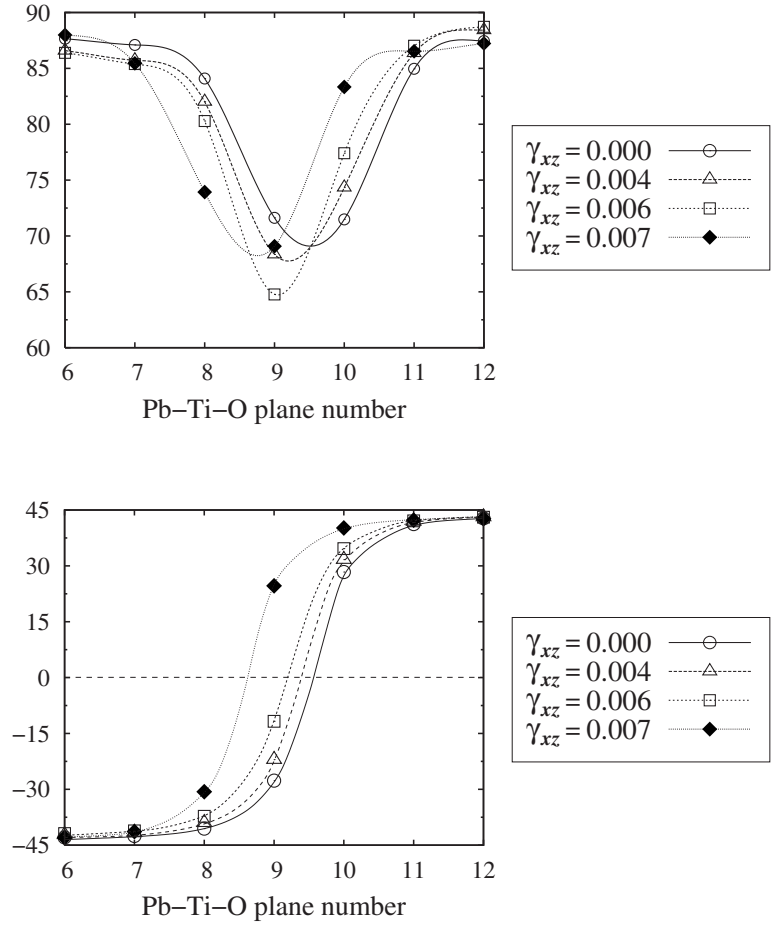

FIG. 6. Change in local polarization distribution across (a) DW-A and (b) DW-B of the simulation cell in $N=18$ under shear deformation. 



FIG. 7. (Color online) Displacements of atoms across (a) DW-A and (b) DW-B at $\gamma_{x z}=0.007$ relative to the unstrained structure in the $N=18$ model. The displacement vectors are enlarged by a factor of 5 for clarity. Black circles, purple triangles, and blue squares are Pb, Ti, and $\mathrm{O}$ atoms, respectively.

magnitude of the shear stress is the same in both cases.

Figure 7 shows the atomic displacements at $\gamma_{x z}=0.007$ from the unstrained structure. Here, the displacement vectors are magnified by a factor of 5 for clarity. The $\mathrm{Pb}$ atoms on the 1 and 9 planes encircled in the figure have large displacements in the $z$ direction. This implies that the displacements of the $\mathrm{Pb}$ atoms trigger the stress-induced domain switching. In $\mathrm{PbTiO}_{3}$, the partially covalent $\mathrm{Pb}-\mathrm{O}$ bond with the hybridization between the $\mathrm{Pb} 6 s$ and the $\mathrm{O} 2 p$ states often plays important roles, e.g., the origin of ferroelectricity, a large tetragonal distortion, ${ }^{22}$ and the reconstruction of the (001) surface and its characteristic responses to in-plane strains. ${ }^{24,25}$ Thus, we focus on the change in the $\mathrm{Pb}-\mathrm{O}$ bonds during the domain switching process. Figure 8 shows changes in the atomistic and electronic configurations of the domain walls projected on the $\mathrm{PbO}(010)$ plane under shear deformation. The $\mathrm{Pb}-\mathrm{O}$ bonds across the domain walls are emphasized by the white lines. Charge density of the $\alpha$ bond on the 1 plane decreases, while that at the $\beta$ site increases, as the shear strain is applied. At $\gamma_{x z}=0.007$, where stressinduced domain switching takes place, the $\alpha$ bond breaks and the $\beta$ bond is newly formed accompanying a large movement of the $\mathrm{Pb}$ atom. Consequently, the convex downward bonding structure of DW-A shifts in the $+x$ direction. A similar mechanism is found in DW-B. The $\alpha^{\prime}$ bond in the 9 plane is weakened with increasing strain, and finally the $\alpha^{\prime}$ bond is reconstructed as the $\beta^{\prime}$ bond. The resulting structure shows the movement of DW-B in the $-x$ direction. Thus, the bond reconstruction from $\alpha\left(\alpha^{\prime}\right)$ to $\beta\left(\beta^{\prime}\right)$ is responsible for stress-induced domain switching.

We make a quantitative comparison between the critical value for polarization reorientation of the single domain
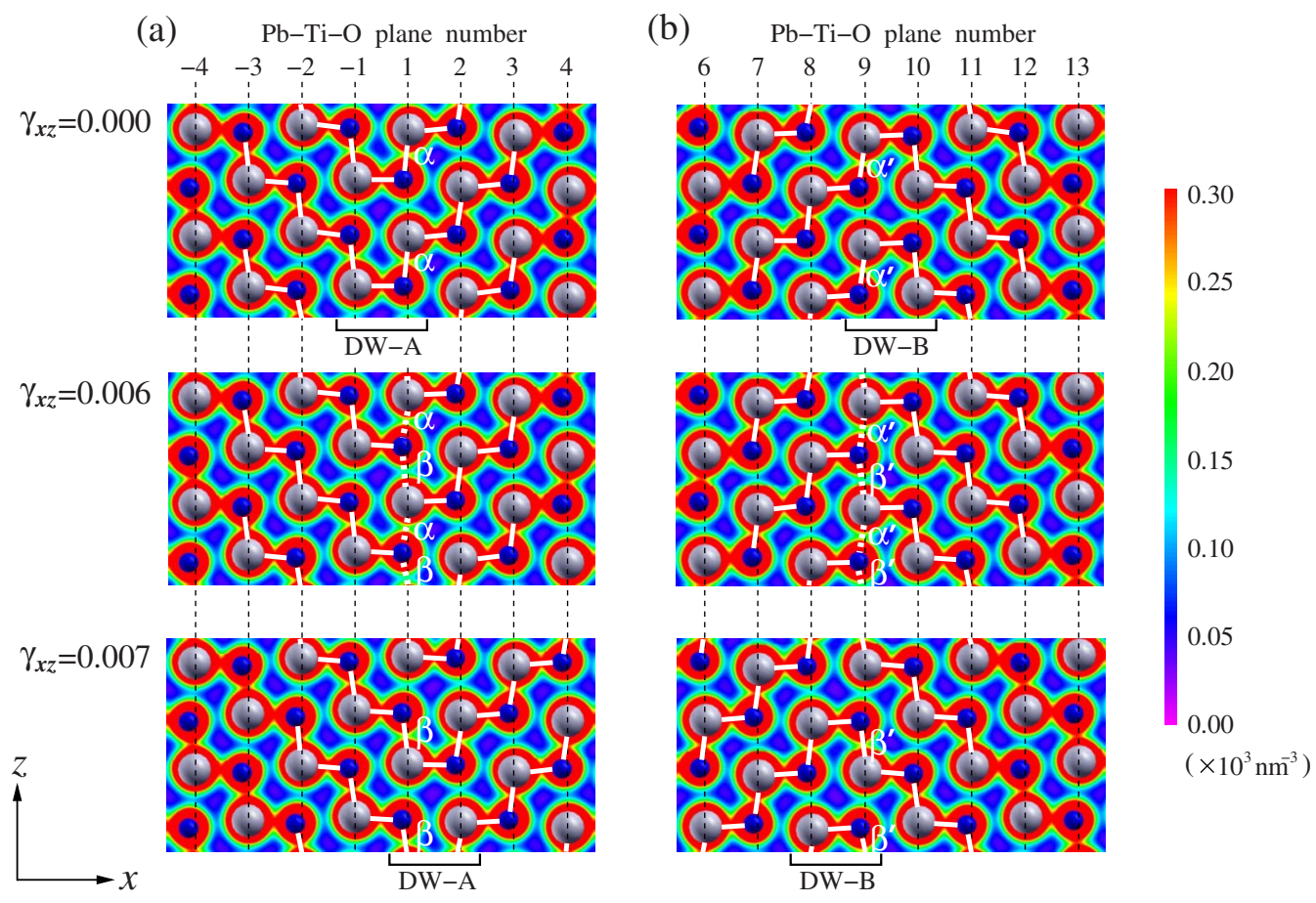

FIG. 8. (Color online) Change in atomistic configurations and charge density distributions near (a) DW-A and (b) DW-B projected on the $\mathrm{PbO}(010)$ plane of the $N=18$ model under shear deformation. Gray and blue spheres are $\mathrm{Pb}$ and $\mathrm{O}$ atoms, respectively. The $\mathrm{Pb}-\mathrm{O}$ bonds around the DWs are drawn with white lines. 


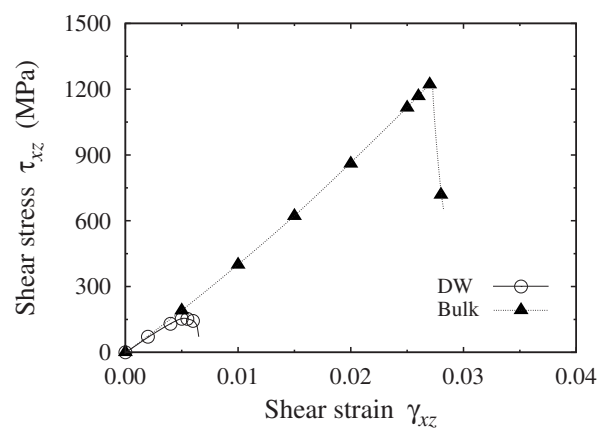

FIG. 9. Shear stress-strain curves of the single domain (bulk) and the domain wall with the $N=18$ model.

(bulk) and the domain wall. For the single domain case, we take the shear direction $\langle\overline{101}\rangle$ on the $\{101\}$ plane of $\langle 001\rangle$ polarized bulk, which corresponds to the shear system of the domain switching. Figure 9 shows the stress-strain curves under shear. The stress in the bulk changes smoothly with increasing strain. When the strain exceeds $\gamma_{x z}=0.027$, the stress in the bulk declines dramatically. The critical shear stress $\tau_{c}$ of the bulk (1221 MPa) is eight times higher than that of the domain switching (152 MPa). This indicates that the polarization rotation through the domain switching is much more favorable than the homogeneous rotation in the bulk. Thus, reorientation of polarization is highly likely to start from the domain wall.

The results presented in this study are for the ideal case of the homogeneous domain switching of a defect-free $90^{\circ}$ domain wall, which is instructive for understanding the fundamental behavior of a domain wall. The calculated critical shear stress of $152 \mathrm{MPa}$ gives an "ideal strength" for stressinduced domain switching. In a real situation, the critical stress can be reduced for several reasons, e.g., because of domain walls with a kink structure. ${ }^{26,27}$ Moreover, there will presumably be a complex effect due to vacancies and/or impurities. In fact, the experimentally observed critical shear stress for a $90^{\circ}$ domain switching in tetragonal $\mathrm{Pb}\left(\mathrm{Mg}_{1 / 3} \mathrm{Nb}_{2 / 3}\right) \mathrm{O}_{3}-\mathrm{PbTiO}_{3}$ is $61 \mathrm{MPa},{ }^{7}$ which is less than half of our result. Thus, investigating such effects is of importance and remains as challenging future work. We believe that our results for the ideal domain wall are helpful as a criterion for the study of the basic influence of kinks, vacancies, and impurities on domain switching.

\section{CONCLUSION}

$A b$ initio density functional theory calculations within the local density approximation have been conducted in order to investigate the atomistic and electronic structure of the $90^{\circ}$ domain wall in $\mathrm{PbTiO}_{3}$ and the fundamental mechanism of shear-induced domain switching. The $90^{\circ}$ domain-wall energy is calculated to be $29 \mathrm{~mJ} / \mathrm{m}^{2}$, which is in good agreement with previous theoretical study. ${ }^{10}$ The magnitude of polarization at the domain wall drops by $20 \%$ from that of the bulk, indicating the suppression of ferroelectricity by the local strain due to the domain wall. We also find a sharp change in the polarization direction within the domain wall where the dominant region is in $1.3 \mathrm{~nm}$. Here, both the series of $\mathrm{Pb}-\mathrm{O}$ and $\mathrm{Ti}-\mathrm{O}$ covalent bonds on the $\mathrm{PbO}$ - and $\mathrm{TiO}_{2}-(010)$ planes are connected smoothly across the domain wall, forming a downward convex of the bonding.

Under shear straining, the deformation is highly localized at the $90^{\circ}$ domain wall. The polarization at the center of the wall gradually changes as the strain increases, and the polarization direction rotates. After the stress reaches the critical magnitude of $152 \mathrm{MPa}$, the domain wall starts to migrate perpendicularly to itself. Here, the propagation direction depends on the shearing direction. During domain switching, a $\mathrm{Pb}-\mathrm{O}$ covalent bond at the center of the domain wall breaks, while another bond on the neighboring $\mathrm{Pb}-\mathrm{O}$ site is concurrently formed with a large movement of the $\mathrm{Pb}$ atom. We conclude that the reconstruction of the $\mathrm{Pb}-\mathrm{O}$ bond is responsible for the domain switching.

Compared with the shear behavior of the single domain (bulk), the $90^{\circ}$ domain wall is much weaker against shear strain: The critical shear stress of the bulk is calculated to be $1221 \mathrm{MPa}$, which is eight times higher than that for domain switching. This result suggests that the reorientation of polarization induced by shear stress is likely to start from the edge of the $90^{\circ}$ domain wall.

\section{ACKNOWLEDGMENTS}

This work was supported in part by the Center of Excellence for Research and Education on Complex Functional Mechanical Systems (COE program of the Ministry of Education, Culture, Sports, Science and Technology, Japan) and by a Grant-in-Aid for Scientific Research (S) (No. 16106002) of the Japan Society of the Promotion of Science. *shimada@cyber.kues.kyoto-u.ac.jp

${ }^{1}$ J. F. Scott, Ferroelectric Memories (Springer, Berlin, 2000).

${ }^{2}$ R. Ramesh, Thin Film Ferroelectric Materials and Devices (Kluwer, Boston, 1997).

${ }^{3}$ M. E. Lines and A. M. Glass, Principles and Applications of Ferroelectrics and Related Materials (Oxford University Press, Oxford, 2001).

${ }^{4}$ W. L. Swartz, IEEE Trans. Electr. Insul. 25, 935 (1990).

${ }^{5}$ D. Damjanovic, Rep. Prog. Phys. 61, 1267 (1998).
${ }^{6}$ E. Jona and G. Shirane, Ferroelectric Crystals (Dover, New York, 1993).

${ }^{7}$ J. K. Shang and X. Tan, Acta Mater. 49, 2993 (2001).

${ }^{8}$ M. Foeth, A. Sfera, P. Stadelmann, and P. A. Buffat, J. Electron Microsc. 48, 717 (1999).

${ }^{9}$ S. Stemmer, S. K. Streiffer, F. Ernst, and M. Rühle, Philos. Mag. A 71, 713 (1995).

${ }^{10}$ B. Meyer and D. Vanderbilt, Phys. Rev. B 65, 104111 (2002).

${ }^{11}$ P. Hohenberg and W. Kohn, Phys. Rev. 136, B864 (1964). 
${ }^{12}$ W. Kohn and L. Sham, Phys. Rev. 140, A1133 (1965).

${ }^{13}$ G. Kresse and J. Hafner, Phys. Rev. B 47, 558 (1993).

${ }^{14}$ G. Kresse and J. Furthmüller, Phys. Rev. B 54, 11169 (1996).

${ }^{15}$ P. E. Blöchl, Phys. Rev. B 50, 17953 (1994).

${ }^{16}$ D. M. Ceperley and B. J. Alder, Phys. Rev. Lett. 45, 566 (1980).

${ }^{17}$ H. J. Monkhorst and J. D. Pack, Phys. Rev. B 13, 5188 (1976).

${ }^{18}$ D. Vanderbilt, Phys. Rev. B 41, 7892 (1990).

${ }^{19}$ R. Resta, J. Phys.: Condens. Matter 12, R107 (2000).

${ }^{20}$ S. Pöykkö and D. J. Chadi, Appl. Phys. Lett. 75, 2830 (1999).

${ }^{21}$ W. Zhong, R. D. King-Smith, and D. Vanderbilt, Phys. Rev. Lett. 72, 3618 (1994)
${ }^{22}$ R. E. Cohen, Nature (London) 358, 136 (1992).

${ }^{23}$ Y. Kuroiwa, S. Aoyagi, A. Sawada, J. Harada, E. Nishibori, M. Takata, and M. Sakata, Phys. Rev. Lett. 87, 217601 (2001).

${ }^{24}$ Y. Umeno, T. Shimada, T. Kitamura, and C. Elsässer, Phys. Rev. B 74, 174111 (2006).

${ }^{25}$ C. Bungaro and K. M. Rabe, Phys. Rev. B 71, 035420 (2005).

${ }^{26}$ S. Yip, Handbook of Materials Modeling (Springer, New York, 2005).

${ }^{27}$ J. M. P. Mato and M. J. Tello, J. Phys. Chem. Solids 44, 1089 (1983). 